Pathologe 2008 • [Suppl 2] 29:214-217

DOI 10.1007/s00292-008-1043-x

Online publiziert:20. August 2008

(c) Springer Medizin Verlag 2008
A. Stege $\mathrm{e}^{1} \cdot$ M. Hummel ${ }^{2}$

${ }^{1}$ Institut für Pathologie, Charité-Universitätsmedizin Berlin, Campus Mitte

${ }^{2}$ Institut für Pathologie, Charité-Universitätsmedizin Berlin, Campus Benjamin Franklin
Das Humane Genomprojekt (HUGO) sowie die voranschreitende Entwicklung von Hochdurchsatzmethoden, die die parallele Analyse einer Vielzahl molekularer Strukturen ermöglichen, revolutionierten das Wissen über die menschlichen Erbsubstanz und seiner Ausprägung. Die Hoffnung der biomedizinischen Forschung ist nun groß, dass diese Erkenntnisse auf molekularer und genetischer Ebene zu neuen Einsichten in die Erkrankungsentstehung und den zugrundeliegenden molekularen Mechanismen führen.

Basis dieser Untersuchungen sind humane Biomaterialien (zumeist Gewebeproben), die im Zuge eines therapeutischen oder diagnostischen Eingriffs entnommen worden sind und nach $\mathrm{Ab}$ schluss der Krankenversorgung nicht mehr benötigt werden. Diese Schlüsselressourcen finden sich in großer Zahl in den Pathologien und lagern als indikationsübergreifende Probenarchive in den dortigen „Kellern“. Im Regelfall nutzen die Pathologien dieses Gewebe für die Qualitätssicherung und Etablierung von Tests in der Diagnostik, für die Lehre sowie für eigene bzw. lokale Forschungsprojekte. Damit sind die Möglichkeiten der Archive jedoch bei Weitem nicht ausgeschöpft. Zukünftig könnte dieses Material in einer standardisierten und umfassenden Bio(material)bank in den Pathologien zusammengefasst werden und in Kombination mit den klinischen Daten wesentlicher Bestandteil der translationalen Forschung sein.

\section{Definition Biobank}

Der Begriff Bio(material)bank (kurz: Biobank) ist ein zurzeit inflationär verwendeter Ausdruck, dem keine einheitliche Definition zugrunde liegt. Möchte man sich aber mit dem Thema näher befassen, so ist vorab eine Begriffsbestimmung bezüglich der Form und Funktion einer Biobank von Nöten. Im Folgenden sollen die Eigenschaften einer Biobank für die biomedizinische Forschung definiert werden.

In der Stellungnahme des Nationalen Ethikrats wird eine Biobank bezeichnet als

„eine Sammlung von Proben menschlicher Körpersubstanzen (z. B. Zellen, Gewebe, Blut und DNA als materieller Träger genetischer Information), die mit personenbezogenen Daten und Informationen ihrer Spender verknüpft sind bzw. werden können. “([1], S. 9)

bezeichnet. Diese allgemeine Definition schließt u. a. Biobanken in der Regenerations- und Transplantationsmedizin sowie der populationsgenetischen oder biomedizinischen Forschung ein, wobei je nach Arbeitsfeld die zugrundeliegenden juristischen, fachlichen und ethischen Rahmenbedingungen differieren.

In der Regenerationsmedizin z. B. wird vitales menschliches Gewebe gesammelt, aufgearbeitet und wieder in den Menschen zurücktransplantiert, wodurch diese Form der Biobank unter das Transplantations- und Gewebegesetz fällt. Eine Biobank hingegen, die sich aus einem Probenarchiv der Pathologie entwickelt hat, archiviert nur avitales Gewebe, welches nicht für den klinischen Einsatz geeignet ist und somit nicht den rechtlichen Grundlagen des Gewebegesetzes unterliegt.

Weitere Differenzierungen können in Hinblick auf die Arbeitsprozesse bzw. die Organisationsformen vorgenommen werden. In diesem Kontext hat die Telematikplattform für medizinische Forschungsnetze (TMF) 4 Modelle entwickelt, die sich u. a. in der Trägerschaft, den Nutzern, der Materialherkunft usw. unterscheiden ([2], S. 3 u. S. 26).

Für die Biobanken, die sich aus den Probenarchiven der Pathologien rekrutieren, ergibt sich jedoch ein elementarer Unterschied, der sie von allen anderen Biobankformen unterscheidet: Die Proben werden nicht im Rahmen eines spezifischen Forschungsschwerpunktes akquiriert, sondern stellen zunächst eine unselektierte Sammlung dar. Erst die (virtuelle) Herauslösung und Überführung der Gewebeproben in einen krankheitsbezogenen Kontext sowie deren Verknüpfung mit patientenbezogenen Daten erfüllt nach Ansicht vieler Experten das Kriterium einer Biobank.

\section{Anforderungen an eine Biobank}

Die zahlreichen Stellungnahmen und Empfehlungen von verschiedenen Institutionen zur Thematik „Biobanken“, die EU-weite Initiative BBMRI („Biobanking and Biomolecular Resources Research Infrastructure") und die zunehmende Zahl 
sich etablierender Biobanken verdeutlichen das gesteigerte wissenschaftliche aber auch politische Interesse. Dieses gestiegene Interesse führte zu einer intensiven Auseinandersetzung bezüglich der rechtlichen und ethischen Aspekte. Dies war und ist auch weiterhin zwingend notwendig, denn bislang liegen noch keine speziellen gesetzlichen Vorgaben zur Verwendung von humanem Gewebe vor. Infolgedessen sieht sich der Forschende/Betreiber einer Biobank einer erheblichen Rechtsunsicherheit ausgesetzt.

Biobanken gelten jedoch nicht nur als Hoffnungsträger für die biomedizinische Forschung, sondern lösen auch vielerorts Befürchtungen aus. Eine unkontrollierte Weitergabe von Proben und Daten könnte zur unberechtigten Verwendung von Informationen zur genetischen Konstellation von Einzelpersonen führen. Dies wiederum könnte negative Auswirkungen auf das berufliche Fortkommen oder auf eine versicherungstechnische Eingruppierung haben. Die Auseinandersetzung mit dieser Problematik ist ebenso wichtig, wie die Lösung logistischer und technischer Fragen (z. B. Standardisierung, Dokumentation und Qualitätsmanagement). Um eine öffentliche Akzeptanz und Vertrauen von und in Biobanken zu erreichen, sind eine transparente Umsetzung, die informierte Einwilligung, die Freiwilligkeit der Spende und ein ausgereiftes Datenschutzkonzept von zentraler Bedeutung.

\section{Informierte Einwilligung (,informed consent ${ }^{\prime)}$ )}

Die informierte Einwilligung („informed consent") stellt ein wichtiges Element dar, um das Persönlichkeitsrecht des Patienten zu wahren und die Rechtmäßigkeit der Verwendung der Proben zu dokumentieren. Es besteht darüber Konsens, dass der Weiterverwendung von diagnostischem Material sowie den klinischen Daten zu Forschungszwecken schriftlich zugestimmt werden muss. Es ist dabei zu beachten, dass neben dem schon erwähnten Persönlichkeitsrecht (Art. 2 Abs. 1 und Art. 1 Abs. 1 Grundgesetz/GG), auch das Eigentumsrecht ( $\$ 953$ Bürgerliches Gesetzbuch/BGB) und das Recht auf Freiheit der Wissenschaft, Forschung und

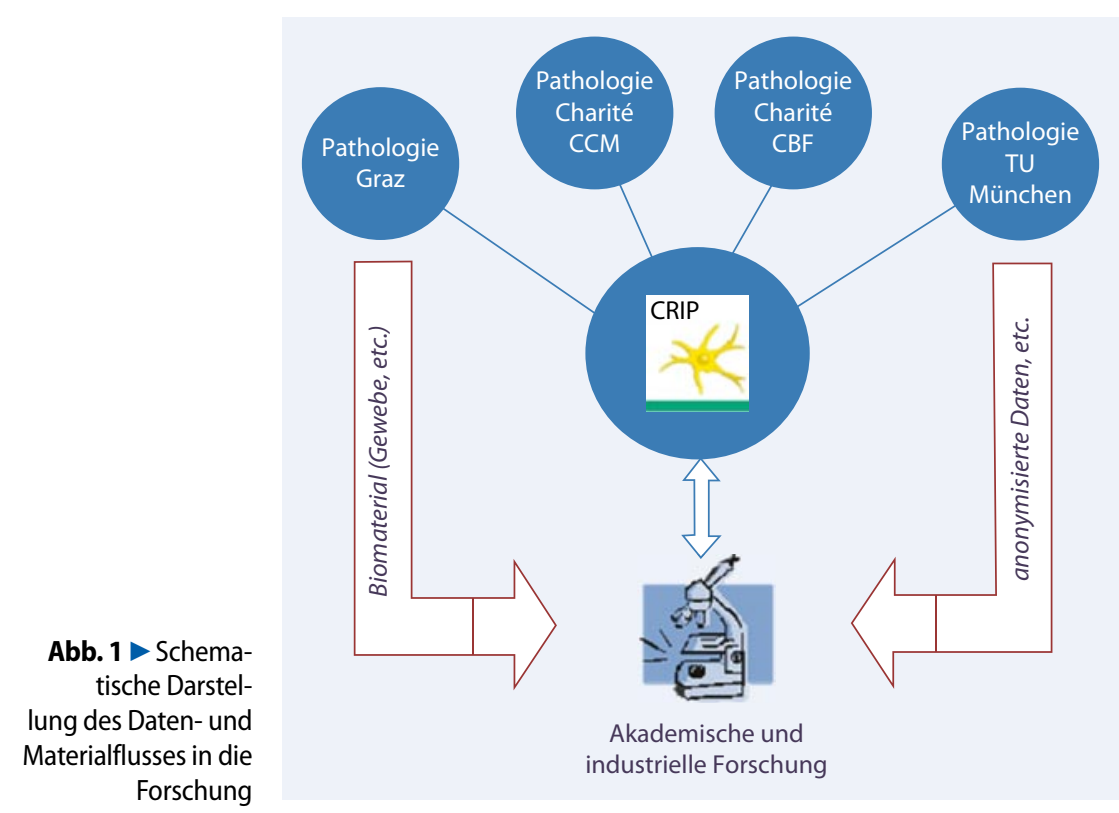

Lehre (Art. 5 Abs. 3 GG) bei dieser Einwilligung berührt werden.

$\mathrm{Zu}$ der Frage, wie eine solche Erklärung aussehen sollte, nimmt der Nationale Ethikrat folgendermaßen Stellung: „Die Spender müssen vor ihrer Einwilligung über alle Umstände aufgeklärt werden, die für ihre Entscheidung über Einwilligung oder Ablehnung erkennbar relevant sein können." ([1], S. 64)

$\mathrm{Zu}$ diesen Umständen gehört zum einen die Erkennbarkeit, dass die Teilnahme auf Freiwilligkeit beruht und keinen Einfluss auf die Qualität der Behandlung hat, und zum anderen, dass ein Widerruf der Zustimmung jederzeit möglich ist (Widerrufsrecht). Der Patient muss ferner über den Zweck, den Umfang und die Dauer der Nutzung ausreichend informiert werden.

Diese nutzungsbezogenen Angaben werden bezüglich ihrer Reichweite, z. B. eine zweckgebundene vs. pauschale Einwilligung, kontrovers diskutiert. Die Nennung des konkreten Forschungszweckes, also die umfangreiche Information des Spenders über den Nutzungsumfang, wird z. T. als Voraussetzung für die Verwendung von Bioproben für die Forschung angesehen ([3], S. 57). Allerdings ist eine strikte Zweckbindung, wie bei klinischen Studien, bei Biobanken in der Regel nicht möglich, da sie als Grundlage für die Durchführung verschiedener Fragestellungen konzipiert werden. Der Na- tionale Ethikrat formuliert den entsprechenden Sachverhalt treffend:

„Wenn vermieden werden soll, dass einmal angelegte Biobanken binnen kurzem entwertet werden, muss die Möglichkeit eingeräumt werden, dass die Spender der Nutzung ihrer Proben und Daten für unbestimmte, erst in der Zukunft zu definierenden Forschungsprojekte zustimmen können. " ([1], S. 59)

Dies kann aber nicht von der Informationspflicht gegenüber dem Spender, der ebenfalls über die zum Zeitpunkt der Einwilligung nicht absehbare Verwendung der Proben aufgeklärt werden muss, entbinden ([2], S. 125 f.).

Während die Verwendung von zukünftigen Biomaterialen mit einer geeigneten Patienteneinwilligung ermöglicht wird, ist die Verwendung von früheren Biomaterialen, die ohne entsprechende Patienteneinwilligung gesammelt wurden, weniger klar geregelt. Eine $-z$. T. geforderte - nachträgliche Einholung einer Patienteneinwilligung ist mit vertretbarem Aufwand nicht möglich, da die Patienten häufig nicht mehr auffindbar oder bereits verstorben sind. Diesen so genannten Altproben kommt jedoch in der Forschung eine besondere Bedeutung zu, da das Material mit einer längeren Krankengeschichte korreliert werden kann, wodurch sich $\mathrm{Pa}$ rameter, die z. B. das Ansprechen auf eine Therapie vorhersagen könnten, identifizieren lassen. Um die Sammlung an 
Pathologe 2008 · [Suppl 2] 29:214-217 DOI 10.1007/s00292-008-1043-x

(c) Springer Medizin Verlag 2008

\section{A. Stege $\cdot$ M. Hummel \\ Erfahrungen bei Einrichtung und Betrieb einer Biobank}

\section{Zusammenfassung}

Das im Rahmen einer diagnostischen oder therapeutischen Intervention entnommene Gewebematerial wird schon seit Jahrzehnten in den Pathologien gesammelt, archiviert und für die lokale Forschung genutzt. Dieses menschliche Restgewebe erlangt jedoch zunehmend das Interesse der biomedizinischen Forschung. Viele Fragestellung, die im Rahmen der so genannten „Translationalen Forschung" aufkommen, können nur mit einer ausreichenden Anzahl an qualitativ hochwertigen Gewebeproben bearbeitet werden. Der besondere wissenschaftliche Wert dieses Materials ergibt sich dabei durch die Verknüpfung mit klinischen Daten.

Biobanken sind aber nicht nur die unentbehrliche Basis der medizinischen Forschung, sondern liegen auch im Spannungsfeld zwischen der Notwendigkeit und dem Schutz der Rechte des Spenders. Mangels gesetz- licher Rahmenbedingungen sieht sich der „Betreibende" oftmals in einer erheblichen Rechtsunsicherheit. Stellungnahmen wie die des Nationalen Ethikrats oder der Telematikplattform für medizinische Forschungsnetze (TMF) geben aber wichtige Impulse für mögliche ethische und rechtliche Lösungen. Unabhängig davon, wie eine zukünftige Lösung aussehen wird, sind die informierte Patienteneinwilligung und ein ausgereiftes Datenschutzkonzept Voraussetzungen für den Aufbau und Betrieb einer Biobank und können darüber hinaus die Bereitschaft der Bevölkerung, ihre Biomaterialen einer Biomaterialbank zur Verfügung zu stellen, erhöhen.

\section{Schlüsselwörter}

Pathologie · Biobanken · Translationale Forschung - Informierte Patienteneinwilligung . Gesetzliche Grundlagen

\section{Experience with establishment and operation of a biobank}

\section{Abstract}

Tissue specimens taken in pathology for diagnostic or therapeutic interventions have been collected for decades, archived and used for local research projects. Meanwhile there is an increasing need of this material for academic and non-academic biomedical research. Many questions in the field of translational research can only be addressed with a sufficient number of high quality tissue samples especially when combined with comprehensive clinical data.

Although biobanks represent an indispensable basis for medical research, this might be in conflict with the rights of patients. Currently there are no specific regula- tions for biobanks leading to substantial legal uncertainties. However, interpretation of existing regulations by the National Ethics Committee or the Telematic Platform for Medical Research Network are important for ethical and legal solutions. Irrespective of future (national or European) regulations, the informed consent of the patients including a secure data protection concept is a prerequisite for establishment and operation of biobanks.

\section{Keywords}

Pathology · Biobanking · Translational research $\cdot$ Informed consent $\cdot$ Legal issues
Altproben zum Wohle der Patienten einsetzen zu können und gleichzeitig einen optimalen Persönlichkeitsschutz für den Spender zu gewährleisten, ist der Nationale Ethikrat der Ansicht, dass die Bedenken gegen die Nutzung ohne Einwilligung am geringsten sind

“..., wenn legitim gewonnene Proben und Daten vollständig anonymisiert wurden, also jeder Personenbezug, der die Identifizierung der Spender erlaubt (Name, Adresse, Geburtsdatum), getilgt wurde. "([1], S. 56)

\section{Datenschutz}

Der wissenschaftliche Wert des Biomaterials ergibt sich erst durch die Verknüpfung mit den krankheitsbezogenen Daten, deren Weiterverwendung aus datenschutzrechtlicher Sicht (Bundesdatenschutzgesetz, BDSG) der Patient ebenfalls zustimmen muss. Dies sollte als integraler Bestandteil der Informierten Einwilligung erfolgen.

Auch die Form der Datenspeicherung/-verarbeitung sowie der Personenkreis, der Zugang zu den Daten hat, muss hinreichend dargelegt werden. Bei der Speicherung und Verarbeitung von Daten ist auf Datensparsamkeit und ein hohes Maß an Sorgfalt zu achten.

Daten einer Biobank können auf 2 Arten gespeichert werden: Zum einen in pseudonymisierter oder in anonymisierter Form. Bei der Pseudonymisierung werden die personenbezogenen Daten durch einen Code ersetzt, wodurch eine Re-Identifizierung des Spenders nur mit Kenntnis des De-Pseudonymisierungscodes möglich wird. Die sichere Verwahrung/Hinterlegung des De-Pseudonymisierungscodes macht eine unzulässige ReIdentifizierung faktisch unmöglich. Die Anonymisierung hingegen führt zu einer völligen und irreversiblen Löschung aller spenderspezifischen Angaben, sodass eine Zuordnung nachträglich nicht mehr erfolgen kann. Welche Form der Codierung gewählt werden sollte, hängt von verschiedenen Faktoren ab (z. B. Patienten-Follow-up). Bei anonymisierten Daten und Proben ist darüber hinaus zu bedenken, dass z. B. ein Widerruf zur Verwendung der Proben durch den Patienten nicht mehr berücksichtigt werden kann. 


\section{Gewebedatenbank CRIP}

An der Charité in Berlin erfolgt zurzeit an beiden Pathologien die Etablierung einer Biobank/Gewebebank, die mit klinischen Daten verknüpft der translationalen akademischen und nichtakademischen Forschung zur Verfügung gestellt werden soll. Für diese Weitergabe von Proben und Daten haben die Ethikkommission der Charité sowie die zuständigen Datenschutzbeauftragten ihre Zustimmung erteilt. Damit können die Altproben - wie vom Nationalen Ethikrat empfohlen - auch ohne nachträgliche Zustimmung der Patienten verwendet werden, während für die zukünftigen Proben eine informierte Patienteneinwilligung vorausgesetzt wird. Die Weitergabe von Daten und Proben an Dritte erfolgt sowohl für die Altproben als auch für die prospektiven Proben nur in anonymisierter Form. Neben den rechtlichen und ethischen Rahmenbedingungen wird für die Akquisition der künftigen Proben ein hohes Maß an Standardisierung angestrebt, das in einem umfassenden Qualitätsmanagement die Integrität der Proben und Daten garantieren soll. Für die Proben aus der Charité-Gewebebank ist eine Aufwandsentschädigung vorgesehen, sodass ein weitgehend kostenneutraler Betrieb erreicht werden soll.

Für die immensen Aufgaben, die auf die biomedizinische Forschung zukommen, kann die Akquisition von Biomaterialien aus einer einzigen Einrichtung nicht mehr ausreichend sein. Die benötigten Probenzahlen, vor allem bei seltenen Erkrankungen, können nicht nur von einer einzelnen Biobank abgedeckt werden. Da es bisher kein Verzeichnis für Biobanken in Deutschland gibt, entstand vor einigen Jahren die Idee, eine Datenplattform zu etablieren, welche dem interessierten Forschenden aus dem universitären und dem industriellen Umfeld die Möglichkeit bietet, in einem großen Datenpool aus mehreren Pathologien nach geeigneten Proben und Forschungspartnern zu suchen.

Basierend auf dieser Idee wurde CRIP ("Central Research Infrastructure for molecular Pathology") ins Leben gerufen. Dieses Internetportal bietet einen Zugriff auf anonymisierte kumulative probenspezifische Daten, die aus den beteiligten Pa- thologien stammen ( $\bullet$ Abb. 1). Eine entsprechende Suchoberfläche (http://www. crip.fraunhofer.de) ermöglicht es dem Anfragenden nach erfolgreicher Registrierung, nach spezifischen Erkrankungen $\mathrm{zu}$ suchen. Werden geeignete Proben für ein Forschungsprojekt in CRIP gefunden, erfolgt im nächsten Schritt eine Präzisierung der Anfrage in direkter Kommunikation mit den beteiligten Pathologien und schließlich eine Kooperationsvereinbarung, im Rahmen derer die Weitergabe der Proben und Daten erfolgt.

\section{Abschlussbetrachtung}

Biobanken spielen eine zentrale Rolle in der Weiterentwicklung der molekularen Medizin. Nur eine große Anzahl von qualitativ hochwertigen Biomaterialen mit den entsprechenden klinischen Daten erlaubt es, zukünftig Risiko- und Prognosefaktoren für Krankheiten zu erkennen und personalisierte Therapien zur entwickeln. Die Pathologien mit ihren umfangreichen Probensammlungen sind dabei ein Garant für einen erfolgreichen Aufbau geeigneter Biobanken.

Auch wenn die Hoffnung und Euphorie groß sind, so müssen neben technischen Fragen, wie Standardisierung der Gewinnung, Lagerung und Aufarbeitung von Probenmaterial, insbesondere die Rechte der Spender berücksichtigt werden. Derzeit gibt es keine spezifischen Gesetze für Biobanken, und es wird intensiv diskutiert, ob die vorhandenen Regelungen nicht doch ausreichen. Stellungnahmen wie die des Nationalen Ethikrats haben wichtige Impulse für mögliche ethische und rechtliche Lösungen gegeben. Unabhängig davon, wie eine zukünftige Lösung aussehen wird, sind die informierte Patienteneinwilligung und ein ausgereiftes Datenschutzkonzept Voraussetzungen für den Aufbau und Betrieb einer Biobank und können darüber hinaus die Bereitschaft der Bevölkerung, ihre Biomaterialen einer Biomaterialbank zur Verfügung zu stellen, erhöhen. Für diese Entwicklung spielen vorhandene und zukünftige Gewebesammlungen in den Pathologien eine zentrale Rolle.

\section{Korrespondenzadresse \\ Dr. A. Stege}

Institut für Pathologie, Charité-Universitätsmedizin Berlin, Campus Mitte

Charitéplatz 1, 10117 Berlin

alexandra.stege@charite.de

Interessenkonflikt. Die korrespondierende Autorin weist auf folgende Beziehung hin: Es besteht eine Kooperation zwischen den Pathologien der Charité und der Gewebedatenbank CRIP.

\section{Literatur}

1. Nationaler Ethikrat (2004) Biobanken für die Forschung - Stellungnahme. http://www.ethikrat. org/themen/pdf/Stellungnahme Biobanken.pdf

2. TMF (Telematikplattform für Medizinische Forschungsnetze) (2006) Bestandsaufnahme und Charakterisierung von Biobanken - Systematisierung wissenschaftliche Bewertung, Finanzierungsmodelle und Konzepte zu Datenschutz und Patienteneinwilligung - Gutachten. Version 1.20. (Autoren: Semler SC, Becker R, Goebel JW et al.). Berlin

3. Wellbrock R (2003) Biobanken - Nutzung menschlicher Zellen und Gewebe. In: Nationaler Ethikrat (Hrsg) Biobanken - Chancen für den wissenschaftlichen Fortschritt oder Ausverkauf der „Ressource" Mensch? Vorträge der Jahrestagung des Nationalen Ethikrates 2002. Berlin 